\title{
Total pressure probe directional scanning imaging principle and device design
}

\author{
Yuankai $\mathrm{Li}^{1, \text { a }}$, Yueming Yang ${ }^{1,}$, Limin Song $^{1, \text { a }}$, Xiujuan Liu $^{1, \text { a }}$, \\ Song $\mathrm{Li}^{1, \mathrm{a}}, \quad$ Xiao $\mathrm{Xu}^{2, b}$, Xuewei $\mathrm{Liu}^{1, \mathrm{a}}$ \\ ${ }^{1}$ Department of Flight Theory, Air Force Aviation University,Changchun,130022, china \\ ${ }^{2}$ Flight Training Base, Air Force Aviation University,Changchun,130051, china \\ aemail: 595883711@qq.com, bemail: ccyym1@126.com,
}

Keywords: total pressure; directional scanning; total pressure probe scanning; vortex structure; color grating; flow layer

\begin{abstract}
: this article from the analysis of total pressure in the flow field in the directional scanning is detected by the pressure signal, this paper discusses the color image technology is used to do high density scanning imaging space complex vortex system. The image has a complete structure, image is clear, the complex vortex system analysis can be strong wait for an advantage, can be used as the study of the production of vortex flow, development and rupture of reliable basis.
\end{abstract}

\section{1 introduction}

Different from general flow display concept, flow field color graphic technology is a kind of flow field parameters of proven, processing and reproduction process, it is the visualization of flow field detection results. This, for the three dimensional flow observation is important, because people want to build from the data in a wide range of empty asked state of complex three-dimensional flow is a difficult thing

Therefore, this kind of method, we could be called "secondary" or "indirect" flow display technology, in order to directly, such as tobacco, helium bubble, hydrogen bubble flow visible display technology of phase difference becomes.

Flow field in the flow field of graphics, color graphic technology had built up with detection means, the flow field in the data processing methods such as closely related. At present, we in the study of the technology developed such a way: with high response characteristics of combined flow field, total pressure probe scanning image detectors circuit was used to extract characteristic values of the parameters such as pressure signal in digital logic circuit and color signal generator to convert these characteristic values for the different color of light signals, with optical fiber transmission is a signal to the pressure measuring point behind, finally through the optical lens imaging in the film, record the result directly. In a recent study, by the means of a model plane for high density around space scanning probe, the display detached vortex structure and the mutual interference between vortex system, good results have been achieved.

\section{2 directional scanning flow field, total pressure probe to detect pressure signal}

Probe detection of flow field directional scanning ways, can improve the detection speed, high density detection on the flow field large range, so as to improve the spatial resolution of the image. At the same time, it is also indicated by detection point location using the signal light, realize the scene shot records required. 
2.1 the flow field in the pitot tube response expression

The response of the flow field in the pitot tube usually can use the following expression:

$P_{S}=P+\frac{1}{2} C \rho V^{2}$

$P_{S}$-The signal of pressure pipe;

$P$-Static pressure of the fluid;

$V$-The speed of the airflow;

$C$-Depends on the tube head shape coefficient.

For spherical, flat end circular and flat rectangular pitot tube, $\theta$ within a certain range,

$C=1-K\left(\sin ^{2} \theta\right)^{m}=1-K\left(V_{n}^{2} / V^{2}\right)^{m}$

In the formula: $\theta$ for nozzle airflow velocity and the Angle between the pipe axis, namely airflow Angle;

$V_{n}=V \sin \theta \quad$-Nozzle air velocity of transverse component

$K$ and $m$ Nozzle geometry size determined by the constant.

Therefore, the above formula can be written as

$P_{S}=P+\frac{1}{2}\left[1-K\left(\sin ^{2} \theta\right)^{m}\right] \rho V^{2}$

That is $P_{S}=P_{s 0}-\frac{K}{2}\left(\sin ^{2} \theta\right)^{m} \rho V^{2}$

In the formula: $P_{S 0}=P+\frac{1}{2} \rho V^{2}$ is when $\theta=0^{\circ}$, The drift Angle is zero pitot pressure, the total pressure.

From $P_{S}=P_{s 0}-\frac{K}{2}\left(\sin ^{2} \theta\right)^{m} \rho V^{2}$,we can see, When the invariable speed, pitot pressure response in the signal, and decreases as the airflow Angle, and is a function of a complex. The pitot tube in practical application, and the relationship is obtained by wind tunnel calibration, and in the form of a calibration curve is given. From total pressure probe used in the structure of the people and the calibration curve, the pressure signal of two surfaces with the change of airflow Angle are basically the same.

2.2 characteristic curve analysis

Curve has the following characteristics worthy of note:

(1) starting from zero Angle,, at a considerable Angle van home, basic does not change with the Angle and total pressure values - color, for a total pressure tube, with training set this Angle can reach more than $40^{\circ}$. On to the end of the garden probes, about $18^{\circ}$, this Angle is called the critical Angle of total pressure tube.

(2) the airflow Angle after more than a critical Angle, total pressure tube measured pressure value will decrease gradually, high real total pressure value is more and more big. Greater than $60^{\circ}$ after that, the curve is very steep inclined, a very close to the slope of the straight line.

(3) studies have demonstrated that in subsonic range, the bushing probe sensitivity of airflow oblique Angle is not basically remain unchanged, that is the size of the critical Angle is independent and flow velocity.

Now, we can look at the total head directional scanning for the flow field of the signal pressure for the following account: 
(1) the flow field, total pressure probe directional scanning signal pressure pipe:

$$
P_{S}=P+\frac{1}{2} \rho V^{2}-\frac{K}{2}\left(\sin ^{2} \theta\right)^{m} \rho V^{2}
$$

In the formula: $P$ - The scanning spot air static pressure;

$$
\frac{1}{2} \rho V^{2} \text { - The point of gas flow pressure; }
$$

$\frac{K}{2}\left(\sin ^{2} \theta\right)^{m} \rho V^{2}$ - Airflow Angle on dynamic pressure of a fixed amount, depending on the specific structure of the pipe.

(2) when the probe point of local airflow Angle is less than the critical Angle, the correction of approximate to zero. At this time:

$$
P_{S}=P+\frac{1}{2} \rho V^{2}=P_{T}
$$

$P_{T}$ - The total pressure.

Therefore, only when scanned points to determine the local airflow direction with the pipe axis Angle is less than the critical Angle, pipe pressure signal can represent the local total pressure values.

(3) in no air viscous flow field in the (such as detached vortex induced velocity field), the total pressure loss. But for in the flow field in the total pressure of directional scanning probe, the pressure signal of value, but will with the size of the flow speed of each point and the direction. This, it is with a total pressure probe directional scanning vortex system can describe all kinds of spiral graphics. Directional scanning vortex system, therefore, to say the total pressure probe, while failing to traffic total pressure value to make accurate measurements, but does not affect the image description of vortex system structure and its influence each other.

\section{3 vortex graphics hierarchy and interfere with each other}

In total pressure probe continuous scanning process, the flow field in the pipe pressure signal is a continuous change of analog. After signal processing, signal pressure be quantified, to record 35 color in the picture. Therefore, continuous distribution parameters in the flow field in the picture is always characterized by a number of different color area. Below we analysis on a picture description of vortex flow, different color area of meaning.

3.1 ideal vortex structure

According to the data [5] [6], swirling motion of fluid machines around the induced velocity field of the space, the available figure five said.

We are concerned about the decision to the size of the pipe pressure signal related parameters of the distribution, they are:

(1), the outflow field in the vortex core in the distribution of tangential velocity $V_{t}$ with $r$ :

In the vortex core: $V_{t}=\frac{\Gamma}{2 \pi R_{0}^{2}} \cdot r$

Outside the vortex core: $V_{t}=\frac{\Gamma}{2 \pi r}$

In the formula: $\Gamma$-Vortex strong 


$$
R_{0} \text {-The vortex core radius }
$$

(2) the outflow field in the vortex core axis to speed $U$ with $r$ the distribution

Outside the vortex core induced velocity field, the velocity, axial velocity is equal to the outside world to namely $U_{\infty}$, that is

$$
U_{r}=U_{\infty}+\int_{r}^{R_{0}}\left(t_{g} \Omega\right) r \cdot d r
$$

$\Omega$ - The spiral Angle of vortex filament movement.

Data [6] give the distribution of vortex velocity $V_{t}$ and axial velocity $U$ of signal.

In addition, the condition is:

(3) outside the nucleus of the induced velocity, the total pressure is certain, for the constant.

$$
P_{t}(r)=P_{t}(\infty)
$$

After the above conditions, we can explain the meaning of the vortex graphics hierarchy.

3.2 vortex graphics hierarchy

By formula (4) in the presence of vortex flow field in a directional scanning signal of the total pressure pipe pressure. Assume that the spiral vortex core direction and wind axis direction are consistent, which consistent with the axis of the scanning probe. So:

$$
P_{S r}=P_{r}+\frac{1}{2} \rho V_{r}^{2}-\frac{K}{2}\left(\sin _{r} \theta\right)^{m} \rho V_{r}^{2}
$$

In the formula:

$r$-Scanning probe points to the center of the vertical vortex center vertical distance; $P_{S r}$-And vortex center from the scanning point, total pressure probe signal pressure; $P_{r}-r$ The local static pressure;

$V_{r}-r$ The local air velocity;

$\theta_{r}-V_{r}$ Formed by jet Angle.

\subsection{1 in the induced velocity field}

On the same circumference of a circle by formula (6), the induced tangential velocity is the same, by formula (7), the axial velocity is the same, equal to $U_{\infty 0}$, therefore, their speed, that is, the local $r$ air velocity is the same:

$$
V_{r}=\sqrt{V_{r}^{2}+U_{\infty}^{2}}
$$

$V_{r}$ The direction of the change in space, but he and direction Angle, i.e. the air current Angle of pipe axis To:

$$
\tan \theta_{r}=\frac{V_{t r}}{U_{\infty}}
$$

Obviously, it is a constant value. 
By formula (10) of the, again with the same radius from the vortex core is at various points in the space has the same value, they must at the same quantitative partition, it presents the same color. Axis and, because of vorticity and wind axis is consistent, that is perpendicular to the detection of surface, therefore these with the colors of the vortex core isometric spot, inevitably composition for, annular color centered on vortex core.

Therefore, we can judge: a color rings on the graphic represents the vortex induced velocity field of a circular flow layer, while the axis of the vortex and axle load, wind graphics is the center of the position of the vortex core.

We'll analyze the meaning of circular hierarchy in graphics. To write (10) is as follows:

$P_{S r}=P_{r}+\frac{1}{2} \rho V_{r}^{2}-\frac{K}{2}\left(\sin _{r} \theta\right)^{m} \rho V_{r}^{2}$

In the induced velocity field, total pressure for a certain value, thus

$P_{S r}=P_{T \infty}+\frac{1}{2} \rho V_{r}^{2}-\frac{K}{2}\left(\sin _{r} \theta\right)^{m} \rho V_{r}^{2}$

When the $r$ decrease and $V_{t r}$ increase (see 6 type), therefore

$\theta_{r}=\tan ^{-1}\left(\frac{V_{t r}}{V_{\infty}}\right), \quad V_{r}^{2}=V_{t r}^{2}+U_{\infty}^{2}$ Both increase, $P_{s r}$ Decreases with the decrease of the $r$.

Therefore, formed a color according to the signal pressure reducing, gradually reduce the annular color of bashan. Each of the same color ring represents a flow layer.

With the "color grating" and "flow layer", the corresponding relations between we can draw the following conclusion:

(1) through the vortex near the surface, the flow layer will be compressed, the color of grating deformation near the surface, level interval variable, vividly showing the influence of compression.

(2) the vortex core axis with the pipe axis, fellowship with symmetrical observation plane no longer flow layer. Tubular flow layer will show the ellipse, the axis of the ellipse indicate the direction of the vortex core axis from the wind axis. The ratio of the long axis of the ellipse short axis and represents the deviation Angle cosine.

(3) vortex in another vortex near by, two vortex induced velocity influence each other, the results also make the deformation flow layer, vortex core position will move, interlayer spacing will also open, through the wash a kind of flow field has a similar change.

From this study can be found:

(1) in a larger strength, just in vitro vortex area, center of graphics rendering a level, even color clutter area. This shows that the change of the pressure signal is very severe. May be: (a) is a vortex core area, its hierarchy is driving the pressure probe burst; (b) is a newly broken vortex core region, are in the midst of dramatic changes in stress distribution; (c) is a actually vortex core area.

All in all, the special area, certainly does not belong to the vortex core outer layer of the induced velocity structure.

(2) after the vortex core broken vortex core layer in the area have a not a circular hierarchy, color distribution more uniform area. We think this is a low pressure area total pressure loss is larger. Where the velocity of still bigger (mainly refers to the axial velocity) pressure is lower, but the direction of air flow throughout has been gradually converge.

\section{4 conclusion}

Graphics on above is reflected in the initial analysis of the vortex flow on along the axial direction for the development of wind change the flow of information provided, there is no study. 
In conclusion, we argue that the total pressure probe directional flow field information obtained by scanning the body vortex system, the content is very rich, image, and the actual flow is very similar, can be used as the study of the production of vortex flow, development and rupture of reliable basis. Could be used as aircraft design reference, has very practical value.

\section{Acknowledgement}

This paper supported by National Natural Science Foundation China (Grant NO:61172126/F010410)

\section{References}

[1] Allen E,Winkelmann. A color video display Tecnnique for Flow Fiedl surveys AIAA [J], 1979.

[2] Koichi Oshima. Computer Aided Flow Visualization.The International symposium on Flow Visualization [C]. Tokyo: DEC.1977.

[3] H.A.Becker,S.A.F.Brown. Response of Pitot proben in turbulent stream. Fluid Mech.vol62 Part 1.1974 .

[4] Xi 'an jiaotong university. Turbine compressor testing technology. 1981.09.

[5] Dictionary editorial board .Aviation industry science and technology dictionary- aerodynamics and Flight mechanics. 1979.

[6] An,Jiguang . aerodynamics and development. Volue12-The first phase 1978.

[7] Yan Chao, Yu Jianjun, Li Junzhe. Research on CFD Scheme and Grid Effect in heat flux [J]. Aerodynamic Transaction of China, Vol.24, 2006.

[8]Chpoun A, Lengrand J C, Heffner K S. Numerical and Experimental Investigation of Rarefied Compression Corner Flow [R]. AIAA 92-2900, 1992.

[9] Boylan D E. Laminar Heat Transfer on Sharp and Blunt Ten-Degree Cones in Conical and Parallel Low Density Flow [C]. Arnold Engineering Development Center, Tullahorna, TN, AEDC-TR-73-106, Aug. 1973

[10]Lockerby D A, Reese J M, Emerson D R, Barber R W. Velocity Boundary Condition at Solid Walls in Rareedgas Calculations [J]. Physical Review E, 70(017303), 2004.

[11] Gokcen T, MacCormack R W. Nonequilibrium Effects for Hypersonic Transitional Flows Using Continuum Approach [R]. AIAA Paper 1989-0461, 1989.

[12] Lockerby D A, Reese J M, Gallis M A. Capturing the Knudsen Layer in Continuum-Fluid Models of Nonequilibrium Gas Flows [J]. AIAA Journal, 43(6), 2005.

[13] Liu Xuewei, Luo Kai. Numerical Simulation on Computational Model of Hypersonic Slip Flow [C]. ICSMMS 2014.

[14] Luo Kai, Pan Sha, Li Hua. Research on Computational Heat Flux Model in Hypersonic Slip Flow [C]. Dynamical Branch of Aerodynamic Academy in China, Chengdu, 2011. 